This document is the accepted manuscript version of the following article:

Tabi, A., Pennekamp, F., Altermatt, F., A7ther, R., Fronhofer, E. A., Horgan, K., ... Saavedra, S. (2020). Species multidimensional effects explain idiosyncratic responses of communities to environmental change. Nature Ecology \& Evolution, 4, 1036-1043. https://doi .org/10.1038/s41559-020-1206-6

\title{
Species multidimensional effects explain idiosyncratic responses of communities to environmental change
}

Andrea Tabi ${ }^{1}$, Frank Pennekamp $^{1}$, Florian Altermatt ${ }^{1,2}$, Roman Alther ${ }^{1,2}$, Emanuel A. Fronhofer ${ }^{1,2,3}$, Katherine Horgan ${ }^{1}$, Elvira Mächler ${ }^{1,2}$, Mikael Pontarp ${ }^{1}$, Owen L. Petchey ${ }^{1}$, Serguei Saavedra ${ }^{4}$

${ }^{1}$ Department of Evolutionary Biology and Environmental Studies, University of Zurich, 190 Winterthurerstrasse, Zurich 8057, Switzerland

${ }^{2}$ Eawag, Swiss Federal Institute of Aquatic Science and Technology, Department of Aquatic Ecology, Überlandstrasse 133, CH-8600 Dübendorf, Switzerland

${ }^{3}$ ISEM, Université de Montpellier, CNRS, IRD, EPHE, Montpellier, France

${ }^{4}$ Department of Civil and Environmental Engineering, MIT, 77 Massachusetts Avenue, Cambridge, Massachusetts 02139, USA 
1 Environmental change can alter species' abundances within communities consistently, e.g., increasing all abundances by the same percentage, or more idiosyncratically. Here, we show how comparing effects of temperature on species grown in isolation and when grown together helps understand how ecological communities more generally respond to environmental change. In particular, we find that the shape of the feasibility domain (the parameter space of carrying capacities compatible with positive species' abundances) helps explain the

composition of experimental microbial communities under changing environmental conditions. We first introduce a measure to quantify the asymmetry of a community's feasibility domain using the column vectors of the corresponding interaction matrix. These column vectors describe the effects each species has on all other species in the community (hereafter referred to as species' multidimensional effects). We show that, as the asymmetry of the feasibility domain increases, the relationship between species' abundance when grown together and when grown in isolation weakens. We then show that microbial communities experiencing different temperature environments exhibit patterns consistent with this theory.

Specifically, communities at warmer temperatures show relatively more asymmetry, and thus the idiosyncrasy of responses is higher when compared to cooler temperatures. These results suggest that while species' interactions are typically defined at the pairwise level, multispecies dynamics can be better understood by focusing on the effects of these interactions at the community level.

Environmental conditions vary through space and time and influence whether ecological communities contain a mix of rare and abundant species or be composed of species with similar biomasses (or abundances) ${ }^{1-3}$. Temperature is one such condition but its effects on different species' biomasses are often inconsistent ${ }^{4}$. While some species can increase in biomass and others decrease as a function of temperature, the same species can also decrease or increase in biomass depending on the presence of other species ${ }^{5-10}$. Importantly, understanding how temperature influences species' performance (i.e., a species' ability to transform external resources into its own biomass) and interactions can provide one approach for explaining such apparently inconsistent effects of temperature ${ }^{5,11}$. Indeed, temperature often alters interactions among plants and animals ${ }^{5}$ and species' interactions can even shift from negative to positive in different temperature environments ${ }^{12-14}$. Mathematical analyses and empirical results show that indirect effects of temperature mediated by species' interactions can be large relative to direct ones ${ }^{15,16}$. Hence understanding how temperature affects species' interactions while at the 
same time accounting for its effects on species' performances has the potential to explain the varied effects of temperature on community composition.

One approach for understanding and predicting effects of temperature on species' performances and on direct interactions is metabolic theory, in which biological rates scale with body size and temperature ${ }^{4}$. Predictions based on metabolic theory often assume common effects of temperature on all species (i.e., one common set of activation energies ${ }^{17-19}$, although variation in the distribution of activation energies can be substantial and skewed $\left.{ }^{20}\right)$. Coupled with the relatively large effects of species' interactions, the effect of temperature on species' growth rates has the potential to create the appearance of idiosyncratic community responses under changing environments, and to explain such variation in effects if understood and accounted for. How temperature affects the distribution of indirect species' interactions is, however, currently quite unclear, as are implications of interaction distributions for species' responses to environmental change. This multidimensional and changing factors have impaired our ability to understand or predict the effect of temperature on population and community dynamics ${ }^{21-23}$.

Here we use a structural approach to investigate why temperature inconsistently affects communities as a function of species' interactions ${ }^{14,24}$. This approach applies a geometric perspective to Lotka-Volterra (LV) models of population dynamics to quantify the domain in the space of carrying capacities compatible with positive species' abundances (the necessary condition for species' coexistence) as a function of species' interactions - what is called the feasibility domain ${ }^{24,25}$. We focus on the effects of temperature on the community composition. We study the effect on community composition by looking at how temperature affects the relationship between species evenness when grown together and the position of species' performance in isolation in the feasibility domain - what we call relative species' performance. We first develop theory to study and measure asymmetry of the feasibility domain using the variability within the column vectors of an interaction matrix. These column vectors describe the effects each species has on all other species in the community, i.e., species' multidimensional effects. Then, we hypothesize that increasing the asymmetry of the feasibility domain decouples species evenness when grown together from their relative performances in isolation. We then present empirical results that corroborate this hypothesis, and that also show how and why temperature have idiosyncratic effects on community responses: the idiosyncratic effects are, in fact, expected by the effect of temperature on the asymmetry of the feasibility domain across communities. 
67

\section{Results}

\section{Theoretical results and predictions}

To establish our hypothesis, first we define with minimum use of mathematics the measures that are used throughout our study (see Box 1 for mathematical details and Figure 1 for a conceptual illustration). In our framework, we consider the performance of a species $i$ in isolation as its carrying capacity $\left(K_{i}\right)$. Note that intrinsic growth rates $\left(r_{i}\right)$ can also be used as a measure of species' performance in isolation, depending on the dynamical model under consideration $^{26,27}$ (see Methods for further details). Then, we consider that a community of species is characterized by an interaction matrix $(\mathbf{A})$, whose elements $\left(a_{i j}\right)$ define the direct per capita effect of a species $j$ on the per capita growth rate of a species $i$. Note that $a_{i j}$ and $a_{j i}$ do not need to be the same. Importantly, the interaction matrix $(\mathbf{A})$ of the community defines the parameter-space region of carrying capacities (or intrinsic growth rates) under which all the species within the community can have positive biomasses at equilibrium $\left(\boldsymbol{N}^{*}>0\right)$. This parameter-space region is known as the feasibility domain $\left(D_{F}(\mathbf{A})\right)^{28}$. The size of the feasibility domain $(\Omega(\mathbf{A}))$ can be calculated by the proportion of such region inside the unit sphere (the L2 norm $)^{25}$ (see Methods for further details). Larger feasibility domains represent larger differences in species' performances (carrying capacities) that are compatible with feasibility.

Assuming that the dynamics of the community are governed by any model topologically equivalent to a $\mathrm{LV}$ model $^{29}$, the location of the vector of carrying capacities observed in monocultures $(\boldsymbol{K})$ inside the feasibility domain determines the specific distribution of species' biomasses at equilibrium within the community ${ }^{27}$. We quantify this distribution by the species evenness $(J \in[0,1])$. Thus, we define the position of species' performance in isolation in the feasibility domain (i.e., the relative performance of species in isolation, $\theta$ ) as the distance between the observed vector of carrying capacities in monocultures and the vector that would result in all species having the same biomass when grown together (i.e., having maximum species evenness). This distance acts as a normalization factor given that only in the case when species do not interact, the vector of carrying capacities $(\boldsymbol{K})$ is exactly proportional to the species' biomasses at equilibrium $\left(\boldsymbol{N}^{*}\right)^{27,30}$ (see Methods for further details).

Note that the geometric centroid of the feasibility domain corresponds to the vector of carrying capacities leading to all species having the same biomass when grown together 27 (maximum species evenness, $J=1)$. This further implies that in order to compare the performance of species across communities, we need to normalize the relative performance $(\theta)$ by the size of the 
feasibility domain as $\theta_{n}=\theta(0.5-\Omega(\mathbf{A}))$, where 0.5 is the maximum size of any feasibility domain $^{25}$ (see Methods for further details). Thus, we estimated the relationship between species evenness when grown together and the relative performance in isolation by the correlation between $J$ and $\Omega_{n}$.

As we previously mentioned, species' interactions $\left(a_{i j}\right)$ can differ in sign as well as strength. Moreover, a community can be characterized by a combination of direct and indirect species' interactions ${ }^{24}$. Thus, to provide a well-defined community-level characterization of species' interactions, we calculate the asymmetry $(\phi(\mathbf{A}))$ of the feasibility domain. Geometrically, this corresponds to the variability across the column vectors (known as spanning vectors ${ }^{25}$ ) of the interaction matrix A. Recall that these columns vectors can be interpreted as the species' multidimensional effects on the community (see Figure 1 for a conceptual representation of these equivalences). Formally, $\phi(\mathbf{A})=\mathrm{SD}\left(\left\|\boldsymbol{v}_{\mathbf{1}}\right\|, \ldots,\left\|\boldsymbol{v}_{\boldsymbol{S}}\right\|\right)$, where SD corresponds to the standard deviation, $\mathbf{v}_{\mathbf{i}}$ is the $i$ th column vector of the interaction matrix $\mathbf{A}$ with $S$ species, and $\|\cdot\|$ corresponds to the $L_{2}$ norm.

Based on the definitions above, we now turn to establish our hypothesis. We hypothesize communities with more symmetric feasibility domains (i.e., small values of $\phi(\mathbf{A})$ ) generate more homogeneous community responses. Among communities, this leads to relative performance in isolation $\left(\theta_{n}\right)$ being tightly correlated with species evenness when grown together $(J)$ (Figure 1C). Otherwise, differences across communities in the asymmetry of the feasibility domain can increase the idiosyncrasy of community responses: weaken any potential association between $\theta_{n}$ and $J$ (Figure $1 \mathrm{~F}$ ). This verbal account of the theory is illustrated with simulations of model communities (see Figures $2 \& 3$ and Methods Section).

How does all this relate to the effects of temperature on community responses? Based on this theory, we can make contingent hypotheses. If temperature has proportionally similar effects on interaction strengths across communities (i.e., if temperature does not affect the asymmetry of the feasibility domains), then temperature will not affect the association of relative performance in isolation and species evenness when grown together (i.e. Figure 1A-C). For example, if temperature doubled the effect on all interactions (including self-regulation), it would not change the shape of the feasibility domain nor its asymmetry. If, however, temperature has different effects on interaction strengths (i.e., temperature increases the asymmetry of the feasibility domain across communities), then temperature will create idiosyncratic community responses (Figure 1D-F), weakening the correlation between $\theta_{n}$ and $J$. 


\section{Empirical results}

We tested these hypotheses against aquatic microbial communities grown in temperature-controlled environments. Each community contained one, two, or three of six species of bacterivorous protists (Colpidium striatum, Dexiostoma campylum, Loxocephalus sp., Paramecium caudatum, Spirostomum teres, and Tetrahymena thermophila) competing for the same food resource (the bacterium Serratia fonticola). Protists, as the most prevalent and diverse organisms on Earth, are essential components of aquatic food webs providing various ecosystem services and also excellent model organisms due to their fast generation and the ease to control experimental conditions ${ }^{31}$. Furthermore, protist growth rates are strongly temperature-dependent ${ }^{32}$, which allows for investigating the effects of different environmental manipulations. Communities experienced either a control temperature $\left(15{ }^{\circ} \mathrm{C}\right)$ which the organisms had already experienced for many generations, or one of five elevated constant temperatures (gradually increasing $2^{\circ} \mathrm{C}$ each level).

At control temperature $\left(15^{\circ} \mathrm{C}\right)$, we observed a negative relationship between relative performance in isolation $\left(\theta_{n}\right)$ and species evenness when grown together $(J)$ in 2 - and 3 -species communities (Figure 4), as expected when the feasibility domains are less asymmetric. These negative relations persisted at 17,19 and $21^{\circ} \mathrm{C}$ for 2 -species communities, and at 17 , and $19^{\circ} \mathrm{C}$ for 3-species communities. Above these temperatures, there was little evidence of a negative relationship, such that relative performance in isolation did not explain species evenness when grown together. Additionally, we found no systematic directional change in the size of the feasibility domain nor the relative performance across temperatures (Figure 5A \& B). Furthermore, and consistent with the theory, these weaker relationships at higher temperatures are accompanied by more asymmetric feasibility domains (Figure 5C). Importantly, these findings reveal that temperature primarily affected species' multidimensional effects on the community, which affected the asymmetry of feasibility domains, which in turn created a weaker relationship between the relative performance $\left(\theta_{n}\right)$ and species evenness $(J)$.

\section{Discussion}

The close match between our empirical findings and our hypotheses corroborates our structural theory of community responses to environmental change. Specifically, the relationship between species evenness when grown together and their relative performance when grown in isolation. This confirms that changes in species' performances due to external perturbations insufficiently explain changes in community composition ${ }^{2}$. Instead, we need to also know the shape 
(asymmetry) of the feasibility domain. Yet, in order to explore the generality of our findings, we need considerably more empirical research examining how temperature, and other environmental factors, affect species' multidimensional effects across communities.

Importantly, our experiment shows that modest increases in temperature do not disrupt the ability of relative performance in isolation to explain species evenness when grown together, but that larger temperature increases do. The observed diversification of community responses appears to be driven by differences in the asymmetry of feasibility domains. This determines a mapping between composition and structural properties that depends on both responses of species' performance and of interactions to environmental change. The increasing asymmetry of feasibility domains with greater temperature change may explain why previous empirical work has shown a lack of unidirectional community responses to warming ${ }^{7}$. Due to the increasing asymmetry of the feasibility domain, species' performance and single pairwise interactions become a less reliable explanatory variable of species evenness when grown together. These results suggest that while species' interactions are typically defined at the pairwise level, multispecies dynamics can be better understood by focusing on the multidimensional nature of these interactions at the community level.

Our results also corroborate theoretical findings on the link between species evenness and productivity $^{27}$ : communities maximize their tolerance to random external perturbation when their compositions are described by a high species evenness and an intermediate level of productivity. This corroboration shows that diversification of species' interactions can be a plausible consequence of different mechanisms responsible for maintaining the tolerance to environmental changes (see Extended Data Figures 3-4). For example, the observed increase in the asymmetry of the feasibility domains is a likely consequence of the multidimensional interaction effect of interspecific variation in thermal sensitivity, differences in thermal range or thermal optima, and differences in adaptation or plasticity to novel temperatures ${ }^{7}$. Importantly, these results suggest that direct and indirect temperature effects are essential to understand (and potentially predict) community dynamics. Indirect effects that complexity brings, whereby change in the abundance of a species affects the abundance of another via a third can be larger compared to direct effects ${ }^{15}$. Our results also suggest that mechanistic models must include the structure of interactions among organisms and not only the direct effects of temperature ${ }^{33}$.

While our theoretical results hold under higher diversity and mechanistic models (see Extended Data Figure 2), in order to move to a general theory of community responses, future experimental work needs to address communities with more than three species and in other 

ecosystems and environments. Such work should explicitly include comparison of theoretical and experimental work, and involve estimation of responses of species' performances and interactions to environmental change. It could also relax some of the assumptions made in our study, such as temporally invariant performances and interaction strengths, and that species' performances are independent of community composition. Also important is to investigate the effects of temporally varying environmental conditions, including increasing variability and extremes in temperature. 
Box 1: Theoretical framework

Species' performance measures the ability of a species to transform resources into its own biomass. This ability depends both on the species' traits and the species' environment. Species' performance is measured as the carrying capacity $\left(K_{i}\right)$ of each species $i$ in isolation or as the intrinsic growth rate $\left(r_{i}\right)$, depending on the mathematical formalism (see Methods). Hereafter, we define all measures below in terms of carrying capacities.

Feasibility domain $\left(D_{F}(\mathbf{A})\right)$ is a community's parameter space comprised by the carrying capacities that provide all species' populations with a positive equilibrium as a function of the interaction matrix A. Formally, under LV dynamics, this feasibility domain corresponds to a convex region defined by $D_{F}(\mathbf{A})=\left\{\boldsymbol{K}=N_{1}^{*} \boldsymbol{v}_{1}+\cdots+N_{S}^{*} \boldsymbol{v}_{S}\right.$, with $\left.N_{1}^{*}>0, \ldots, N_{S}^{*}>0\right\}$, where $\boldsymbol{N}^{*}$ are the positive solutions of the system, $\boldsymbol{v}_{\boldsymbol{i}}$ are the column vectors of the interaction matrix $\mathbf{A}$, and $S$ is the number of species in the community. The column vectors of an interaction matrix can be ecologically interpreted as the multidimensional interaction effects of an individual species on the community. Recall that the elements $\left(a_{i j}\right)$ of the interaction matrix (A) define the direct per capita effect of a species $j$ on the per capita growth rate of a species $i$.

Geometric centroid of the feasibility domain $\left(\boldsymbol{K}_{\boldsymbol{c}}\right)$ corresponds to the point of maximum species evenness whenever the columns of the interaction matrix have been normalized under any norm ${ }^{25}$. This is true given that in this case, the centroid is equivalent to the center of mass of a convex object with $n$ vertices all having the same mass. Formally, the centroid is calculated as $\boldsymbol{K}_{\boldsymbol{c}}=\frac{1}{S} \boldsymbol{v}_{1}+\cdots+\frac{1}{S} \boldsymbol{v}_{S}$ which corresponds to the conditions under all species have the same biomass at equilibrium.

Species evenness $(J)$ is a description of the distribution of species biomasses within a community. Formally, it is defined as $J\left(N^{*}\right)=-\sum_{i=1}^{S} P_{i} \log \left(P_{i}\right) / \log (S) \in[0,1]$, where $P_{i}$ is the relative biomass of species $i$ at equilibrium, i.e., $P_{i}=N_{i}^{*} / \sum_{j}^{S} N_{j}^{*}$. Note that $J\left(N^{*}\right)=1$ is the case when all species have the same biomass.

Asymmetry of the feasibility domain $(\phi(\mathbf{A}))$ is the variation across all the column vectors of an interaction matrix $\mathbf{A}$. Note that the column vectors correspond to the spanning vectors of the feasibility domain $D_{F}(\mathbf{A})$ ), implying that $\phi(\mathbf{A})$ represents geometrically the asymmetry of the feasibility domain. Mathematically, it is given by $\phi(\mathbf{A})=\operatorname{SD}\left(\left\|\boldsymbol{v}_{\mathbf{1}}\right\|, \ldots,\left\|\boldsymbol{v}_{\boldsymbol{S}}\right\|\right)$, where SD corresponds to the standard deviation and $\|\cdot\|$ corresponds to the $L_{2}$ norm. The higher the value of $\phi(\mathbf{A})$, the more asymmetric the feasibility domain.

Relative performance in isolation $(\theta)$ is defined as the distance between the vector of carrying capacities observed in monoculture $(\boldsymbol{K})$ and the vector of carrying capacities that would result in all species having the same biomass when grown together (i.e., the geometric centroid $\left(\boldsymbol{K}_{\boldsymbol{c}}\right)$ of the feasibility domain). Simply put, this measure captures the position of performances in isolation in the feasibility domain. Formally, it is measured as $\theta=\arccos \left(\frac{\boldsymbol{K} \cdot \boldsymbol{K}_{c}}{\|\boldsymbol{K}\| \cdot\left\|\boldsymbol{K}_{c}\right\|}\right)$, where $\|\cdot\|$ corresponds to the $L_{2}$ norm. Note that this distance normalizes species' performances by the interaction matrix $\mathbf{A}$, given that the geometric centroid $\left(\boldsymbol{K}_{\boldsymbol{c}}\right)$ is particular of every interaction matrix.

Size of the feasibility domain $(\Omega(\mathbf{A}))$ is the proportion of the unit sphere of carrying capacities that provide positive equilibria for all populations in the community. That is, the size corresponds to the normalized solid angle generated by the feasibility domain $D_{F}(\mathbf{A})$, such that it is equal to one for the whole unit sphere $\mathbb{B}^{S}$. The normalized solid angle $\Omega(\mathbb{A})$ is equal to the probability of sampling uniformly a vector of carrying capacities on the unit sphere inside the feasibility domain of an interaction matrix $\mathbb{A}$. Formally, it is calculated as $\Omega(\mathbf{A})=\frac{\operatorname{vol}\left(D_{F}(A) \cap \mathbb{B}^{S}\right)}{\operatorname{vol}\left(\mathbb{B}^{S}\right)} \in[0,0.5]^{25}$. 
A

Homogenous interaction

a

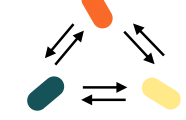

b

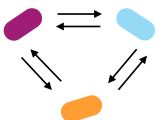

D

c

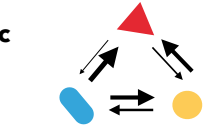

d

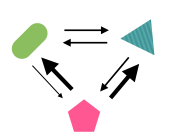

B

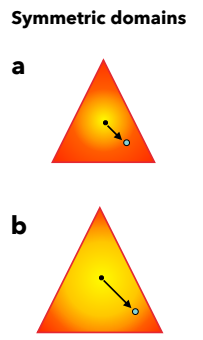

$\mathbf{E}$

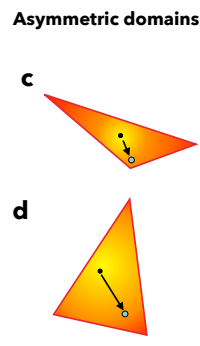

C

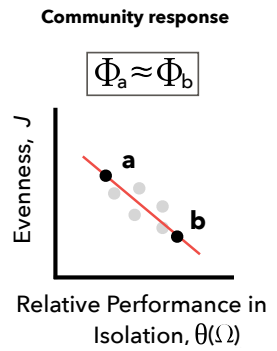

$\mathbf{F}$
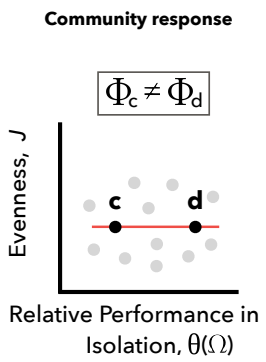

$$
\theta \rightarrow \quad \boldsymbol{K} \circ \quad \Omega \quad \Delta \quad J_{1}
$$

Figure 1: Theory relating differences in species' performances in isolation and species evenness when grown together. Hypothetical 3-species communities in which symbols and colors correspond to different species, while the thickness of arrows represents the direct pairwise interaction strengths. Communities $a$ and $b$ have more variability in the strength of species' interactions and communities $c$ and $d$ have less variability. The triangles in panel B \& E show a 2-dimensional simplex (projection) of a 3 -dimensional cone generated by the column vectors of the interaction matrix. This simplex corresponds to the feasibility domain - region encapsulating all the vectors of the carrying capacities $\boldsymbol{K}$ (or intrinsic growth rates, $\boldsymbol{r}$ ) leading to positive biomasses at equilibrium (see Box 1 for further details). The yellow and red areas inside the feasibility domain represent higher and lower levels of species evenness $J$, respectively. The size and asymmetry of the feasibility domain are represented by $\Omega$ and $\phi$, respectively. Note that the distribution of species' biomasses has maximum evenness $(J=1)$ at the centroid of the feasibility domain (black circle). Instead, the corner defines the location of perfect unevenness $(J=0)$, whereas at the border one has partial unevenness. The blue circle inside a feasibility domain corresponds to the vector of carrying capacities observed in monocultures, $\boldsymbol{K}$. The arrows show the distance between the observed vector $\boldsymbol{K}$ (or $\boldsymbol{r}$ ) and the centroid of the feasibility domain. We call this distance the relative performance in isolation $(\theta)$. Top row: a scenario in which different communities have homogeneous interactions (Panel $\mathbf{A}$ ), which can be translated into a symmetric feasibility domain (Panel $\mathbf{B}$ ), which leads to a strong negative relationship between species evenness when grown together (as a measure of the distribution of species biomasses) and the relative performance in isolation (Panel $\mathbf{C}$ ). Bottom row: a scenario in which communities have heterogeneous interaction (Panel D) that result in asymmetric feasibility domains (Panel $\mathbf{E}$ ), which lead to an unpredictable outcome between species evenness and relative performance in isolation (Panel $\mathbf{F})$. 


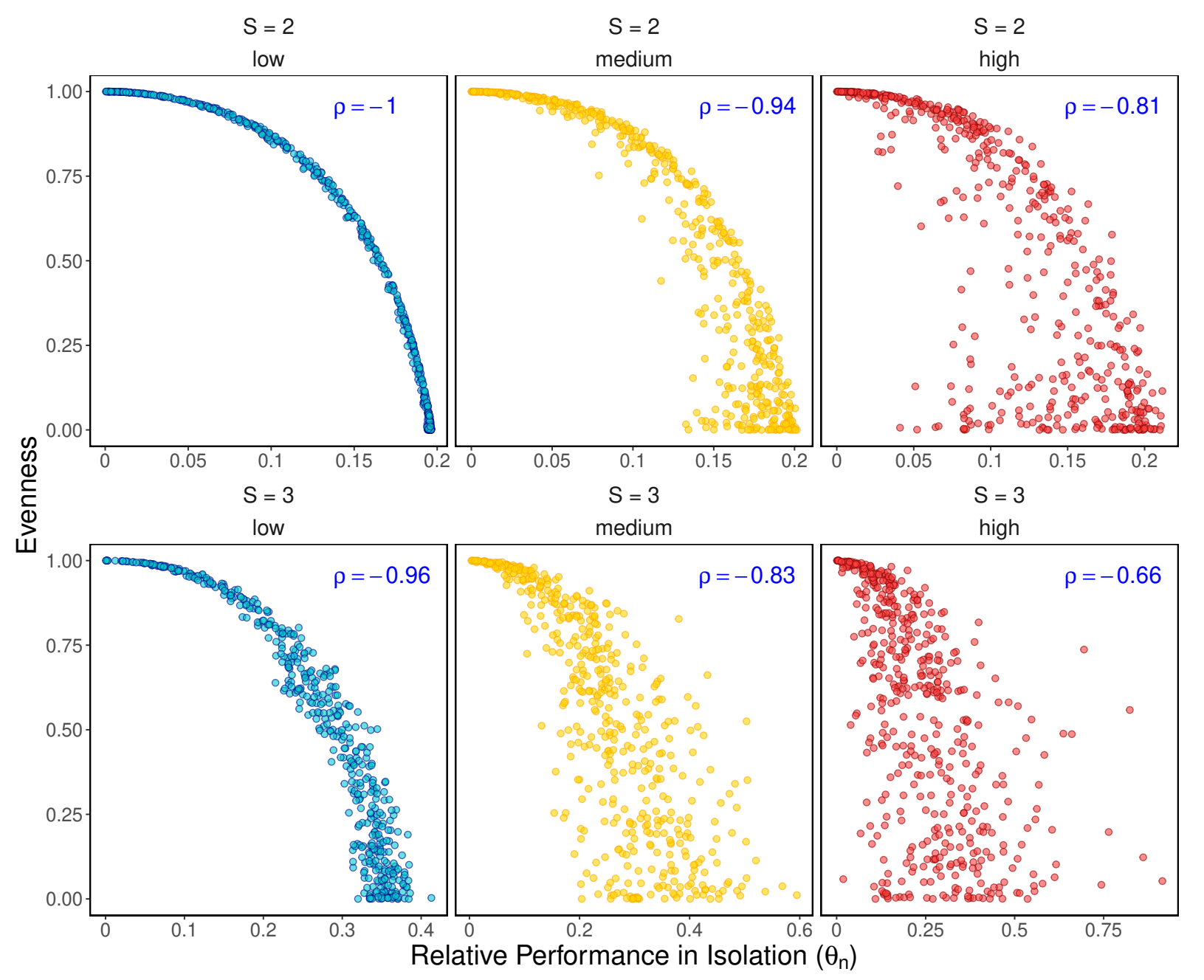

Figure 2: Theoretical results. Each point $(n=500)$ represents a different model-generated community with two species (top row) or three species (bottom row) under different asymmetry values of feasibility domains $\phi(\mathbf{A})$ (low $=0.1$, medium $=0.5$ and high $=0.9)$ marked at the top of each panel. We calculated species evenness at equilibrium $\left(J\left(\boldsymbol{N}^{*}\right)\right)$ and the relative performance in isolation $\left(\theta_{n}\right)$. We also report the Spearman's rank correlation coefficients $(\rho)$, all p-values are $<0.001$. See Methods for full details. 

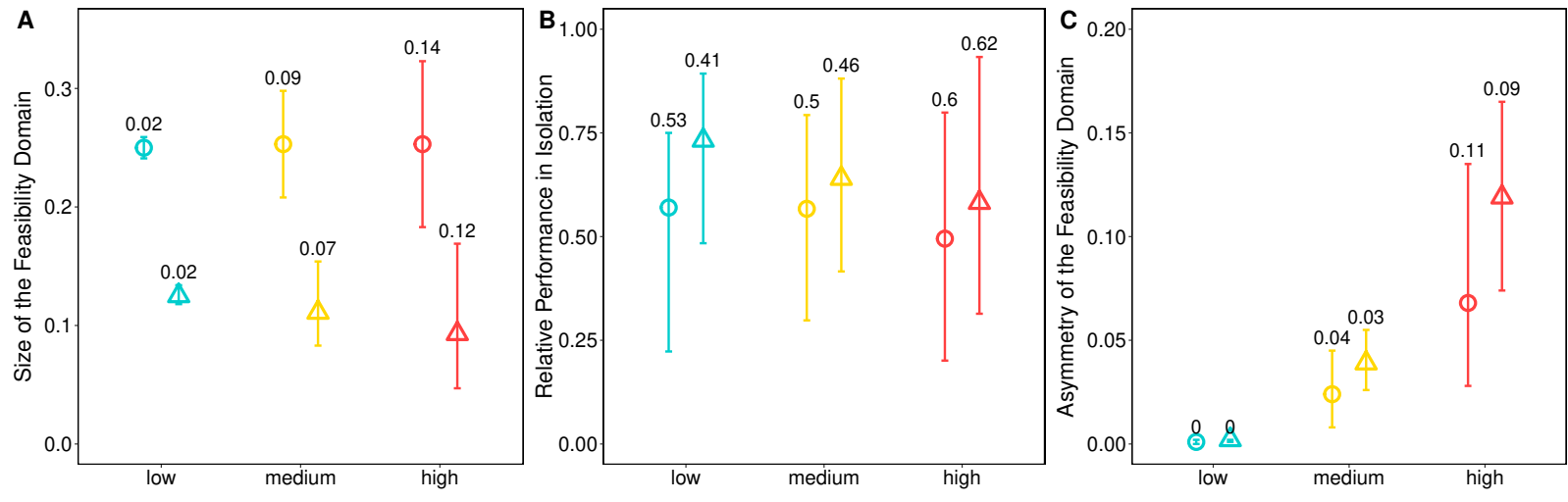

Level of Asymmetry in the Feasibility Domain

Figure 3: Theoretical distribution of structural measures. Circles and triangles represent the median values of 2 - and 3 -species model-generated communities, respectively. The x-axes show different asymmetry values of feasibility domains. All communities are characterized by randomlygenerated interaction matrices using a normal distribution with zero mean and different values of standard deviations which were drawn from a uniform distribution ranging between $0.1,1$, and 10. For each model-generated community and level of standard deviation, we sampled a random vector of carrying capacities $\boldsymbol{K}$ within its size of feasibility domain $\Omega$. The interquartile ranges are shown on the top of each intervals. Panels (A-C) correspond to the size of the feasibility domain $(\Omega)$, the relative performance in isolation $\left(\theta_{n}\right)$, and the asymmetry of the feasibility domain $(\phi)$, respectively. See Methods for full details. 


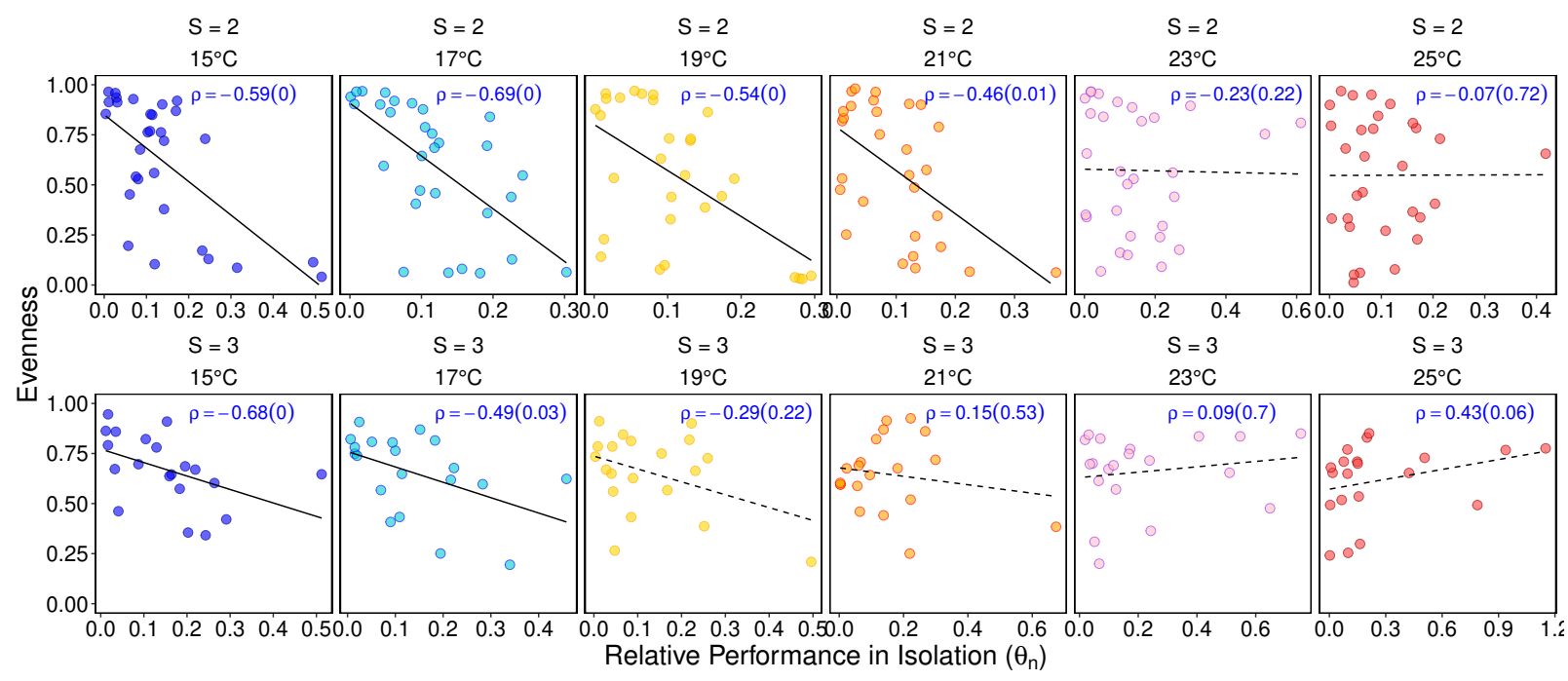

Figure 4: Empirical results. This figure shows experimental microbial communities formed by different combinations of $2(\mathbf{A})$ and $3(\mathbf{B})$ protist species under different temperatures. The first column corresponds to communities under a control temperature of $15{ }^{\circ} \mathrm{C}$, whereas the other columns correspond to the communities at elevated constant temperatures. Panels show the relationship between the observed species evenness $(J)$ and the inferred relative performance in isolation $\left(\theta_{n}\right)$. Inside the panels, we report the Spearman's rank correlation coefficients $(\rho)$ with corresponding p-values inside parentheses among all experimentally-generated communities. Solid and dotted lines correspond to slopes that are statistically distinguishable and non-distinguishable from zero, respectively. See Methods for full details.

O2-species $\Delta$ 3-species
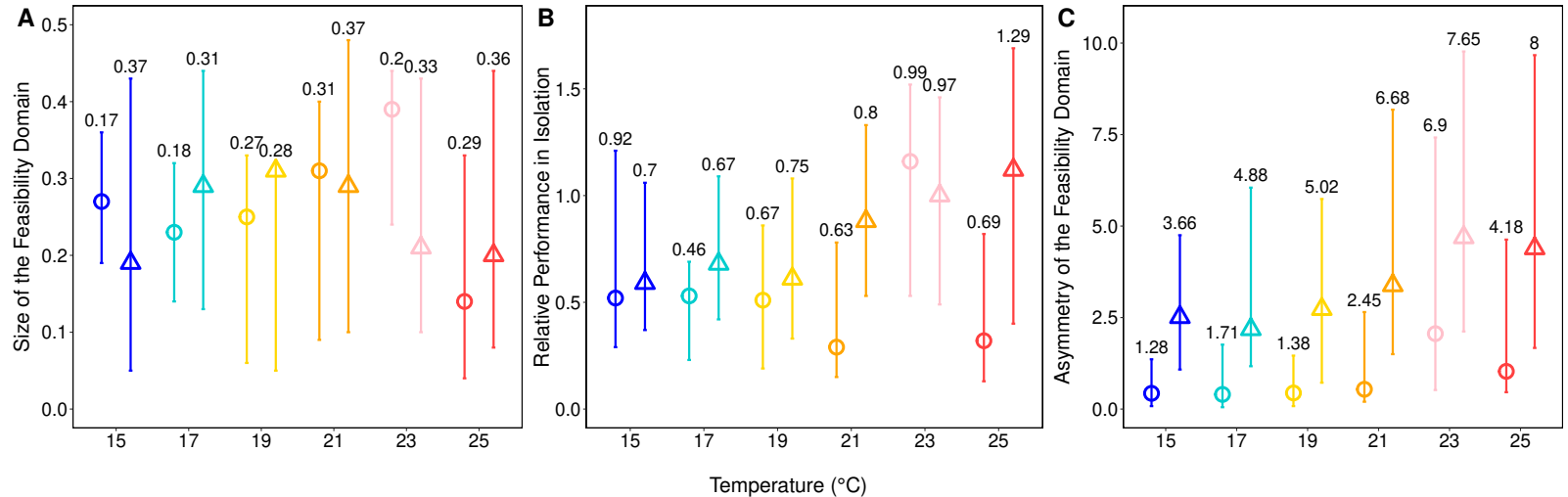

Figure 5: Empirical distribution of structural measures across temperatures. Distribution of the size of the feasibility domain $(\Omega)$, relative performance in isolation $\left(\theta_{n}\right)$ and asymmetry of the the feasibility domain $(\phi)$ across temperatures. Circles (2-species communities) and triangles (3-species communities) denote the median of the corresponding measures calculated from the observations and the error bars denote the 0.25 and 0.75 quartiles obtained from bootstrapping. The numbers on error bars show the magnitude of the interquartile range. See Methods for full details. 


\section{Methods}

\section{Theory and simulations}

For our theoretical investigation, we defined the population dynamics given by the classic Lotka-Volterra (LV) model using the K-formalism $\dot{N}_{i}=N_{i} \frac{r_{i}}{K_{i}}\left(K_{i}-\sum_{j=1}^{S} a_{i j} N_{j}\right)$, where $N_{i}$ is the biomass of species $i, r_{i}$ is the intrinsic growth rate of species $i$, and $a_{i j}$ is the direct per capita effect of species $j$ on $i$. The biomasses at equilibrium are calculated as $\boldsymbol{N}^{*}=\mathbf{A}^{-1} \boldsymbol{K}$. Note that the carrying capacity of species $i$ is defined as $K_{i}=r_{i} / \alpha_{i i}$. That is, the model can be written in the r-formalism as $\dot{N}_{i}=N_{i}\left(r_{i}-\sum_{j=1}^{S} \alpha_{i j} N_{j}\right)$, where $\boldsymbol{N}^{*}=\boldsymbol{\alpha}^{-1} \boldsymbol{r}$. That is, in the K-formalism, the carrying capacities modulate the equilibrium points, whereas in the r-formalism is the intrinsic growth rates which determine the equilibrium points. Note, however, that $\mathbf{A}$ and $\boldsymbol{\alpha}$ do not have the same units. Here we used the K-formalism to illustrate our work; however, both formalisms are interchangeable for our purposes and their use should depend on data availability.

Recent work ${ }^{29}$ has shown that in any model topologically equivalent to the LV model, the structure of species interactions (embedded in the interaction matrix $\mathbf{A}$ ) defines a unique relationship between parameters $\boldsymbol{K}$ and the community composition at equilibrium $\boldsymbol{N}^{*}$ (where $\dot{N}=0$ ). This relationship is established by the feasibility domain, which corresponds to a convex region $D_{F}(\mathbf{A})$ within the parameter space, from which is possible to link uniquely a set of $K_{i}$ to a set of feasible (positive) solutions $N_{i}^{*}>0$ (see Box 1 for further details). Formally, this feasibility domain can be written as $D_{F}(\mathbb{A})=\left\{\boldsymbol{K}=N_{1}^{*} \boldsymbol{v}_{1}+\cdots+N_{S}^{*} \boldsymbol{v}_{S}\right.$, with $\left.N_{1}^{*}>0, \ldots, N_{S}^{*}>0\right\}$, where $\boldsymbol{N}^{*}$ are the positive solutions of the system, $\boldsymbol{v}_{\boldsymbol{i}}$ are the column vectors of the interaction matrix $\mathbf{A}$, and $S$ is the number of species in the community. This definition implies that the feasibility domain of an interaction matrix can be geometrically represented as an algebraic cone by normalizing the parameter space under any norm ${ }^{25}$. An algebraic cone is defined as the space spanned by positive linear combinations of S linearly independent vectors $\boldsymbol{v}_{\boldsymbol{i}}$. Then, the size of the feasibility domain can be estimated by normalizing the solid angle generated by the feasibility domain, such that it is equal to one for the whole unit sphere (using the L2 norm) $\mathbb{B}^{S}$. This normalized angle can be analytically calculated by $\Omega=\frac{1}{(2 \pi)^{S / 2} \sqrt{|\operatorname{det}(\alpha)|}} \int \cdots \int_{N^{*} \geq 0} e^{-\frac{1}{2} N^{* \mathrm{~T}} \alpha^{\mathrm{T}} \alpha N^{*}} \mathrm{~d} N^{*}$, and is computed via a quasi-Monte Carlo method ${ }^{24,34}$.

To theoretically investigate the relationship between species evenness and the relative performance in isolation, we generated 2- and 3-species communities by randomly sampling interaction matrices following a uniform distribution $U[-P, P]$. We used a tuning parameter 
$(\mathrm{P})$, where the larger the values of $\mathrm{P}$, the larger the asymmetry of the interaction matrix is. $\mathrm{By}$ including positive and negative interaction coefficients, we ensured the comparability to our empirical results. All intra-specific coefficients are set to $a_{i i}=-1$, such that each species saturates to its carrying capacity in isolation. This is an important consideration to take into account given that if one aims to change all pairwise interactions in a community, these values would have to be normalized such that the diagonal elements are always equal to one. Our results are qualitatively robust to the choice of distribution ${ }^{34}$. We assumed a fully-connected interaction structure for both 2- and 3-species communities (i.e. connectance is 1).

Parameterizations of $K_{i}$ inside the feasibility domain are sampled by $K_{i}=\sum_{j=1}^{S} N_{i}^{*} v_{i}$, where $N_{i}^{*}$ are all values in $(0,1)$ and $\sum_{i=1}^{S} N_{i}^{*}=1$.

We then calculated the size of the feasibility domain $(\Omega)$, relative performance in isolation $\theta$, the asymmetry of the feasibility domain $\phi$, and the species evenness $J\left(N^{*}\right)$ of the randomly-generated communities (see Box 1 for definitions). We studied how species evenness $J\left(\boldsymbol{N}^{*}\right)$ changed as a function of the relative performance in isolation $\theta$ across different values of asymmetry. Figure 3 confirms that the higher the asymmetry, the higher the variation (measured as the interquartile range) of $\Omega, \theta$, and $\phi$ across communities. Additionally, regardless of the asymmetry, $\Omega$ and $\theta$ were positively correlated, while $\phi$ was not correlated with any measure. This confirmed that the relative performance in isolation needs to be normalized by the size of the feasibility domain in order to be compared across communities: we normalized it as $\theta_{n}=\theta(0.5-\Omega)$ (note that 0.5 is the least upper bound of $\left.\Omega\right)^{25}$. In turn, Figure 2 confirms that $J\left(\boldsymbol{N}^{*}\right)$ and $\theta_{n}$ are negatively correlated under low asymmetry. However, the higher the asymmetry, the more the relationship between $J\left(\boldsymbol{N}^{*}\right)$ and $\theta_{n}$ weakens, indicating that the relative performance in isolation becomes less and less a reliable indicator of species evenness. Importantly, these differences are driven by the asymmetry of the feasibility domains $\phi$. Importantly, the asymmetry is size dependent and can be modulated by the structure of a community, e.g., changing the connectance within a community. Yet, the effect of asymmetry on the relationship between relative performance in isolation and species evenness when grown together remains (see Extended Data Figure 2).

\section{Empirical methods}

We factorially manipulated temperature $\left(15,17,19,21,23\right.$ and $\left.25^{\circ} \mathrm{C}\right)$ and community composition (31 unique compositions). Each of the six temperature treatments was controlled by two independent incubators. Prior testing showed low temperature variation of the liquid medium (set-point temperature varied by $0.1^{\circ} \mathrm{C}$ ). Measuring temperature with a replicated 
gradient is recommended to harness the power of a regression design, while still allowing to test for a nonlinear temperature effect ${ }^{35}$. Long-term protist cultures are kept at $15^{\circ} \mathrm{C}$, representing the control temperature to which the species used in the experiment are adapted. Warming usually decreases their carrying capacities but increases growth rates ${ }^{36}$. Experimental communities were created by growing protists to their respective carrying capacities at $20^{\circ} \mathrm{C}$ in 1L of bacterized medium. The medium consisted of protist pellets (Carolina Biological Supplies, Burlington, NC, USA) at a concentration of $0.055 \mathrm{gL}^{-1}$ of Chalkley's medium in which the bacterium Serratia fonticola was grown as common resource for the bactivorous protists. Two autoclaved wheat seeds were added to each bottle for slow nutrient release. Monocultures were initiated at a density of 3 individuals $\mathrm{mL}^{-1}$ in a total of $100 \mathrm{~mL}$ medium. Communities were initiated with a total of $40 \mathrm{~mL}$ protist culture topped up with $60 \mathrm{~mL}$ fresh medium $(100 \mathrm{~mL}$ culture in total). The $40 \mathrm{~mL}$ culture were assembled by adding a fixed fraction (i.e. $20 \mathrm{~mL}$ for two species, 13.33 for three species) of each species at their specific carrying capacity, adopting a substitutive design. Each experimental community was cultivated in $250 \mathrm{~mL}$ Duran bottle. Since the number of possible species compositions exceeded the number of feasible experimental units, we used all possible compositions only for the monocultures (6 compositions, 3 replicates) and two species communities (15 compositions, 2 replicates). For three species communities, ten compositions (2 replicates) were randomly selected from the set of all possible compositions such that all species occurred the same number of times. This generated a total of 68 experimental units per temperature. Microcosms were sampled 19 times over 36 days to measure community dynamics. To do so, a microcosm was taken out of the incubator, gently stirred to homogenize the culture, and a fixed sample pipetted into a counting chamber. The height of the sampling chamber was $600 \mu \mathrm{m}$ and the area filmed $68.7 \mathrm{~mm}^{2}$ resulting in $41.2 \mu \mathrm{L}$ sampled. The counting chamber was covered with a lid and a 5 second video was taken under the microscope. The videos were subsequently processed with the $\mathrm{R}$ package BEMOVI ${ }^{37}$ to extract morphological and behavioural traits. Individuals in polycultures were classified into species by a random forest classifier trained on trait information obtained from the monoculture data $^{38}$. We derived the biomass of each species by summing the biovolume of all individuals of a given species in a given community and multiplying biovolume with a constant density equal to water (i.e. $1 \mathrm{~g} / \mathrm{cm}^{3}$ ).

\section{Estimation of species interactions}

We fitted a topologically equivalent model to the classic LV model ${ }^{29}$ to our observations using the following form $\dot{N}_{i}=N_{i} \frac{r_{i}}{K_{i}}\left(K_{i}-\sum_{j=1}^{S} a_{i j} \frac{2 N_{j}}{\left(1+N_{j}^{\beta}\right)}\right)$, where $\beta$ is a tuning parameter that allows us to gradually enter more nonlinear forms of functional responses $(\beta \in[0,2]$ by step size 0.1$)$. 
Note that $\beta=0$ results in a linear functional response. These models were fitted (see an example in Extended Data Figure 1) to 178 out of 180 combinations (due to early extinctions) where all possible pair combinations were represented (composition (15) x temperature (6) x replicate (2)) and to 120 three-species communities where also all possible species pairs were contained (not all possible three-way combinations). The model parameters (carrying capacities $K_{i}$ and growth rates $r_{i}$ ) were obtained by fitting logistic growth models to 36 monoculture time series using the following form: $\dot{N}_{i}=N_{i} \frac{r_{i}}{K_{i}}\left(K_{i}-\frac{2 N_{i}}{\left(1+N_{i}^{\beta}\right)}\right)$. Growth rates were fitted to the average biomass (of three replicates) at each time point. Carrying capacities were calculated as the median biomass from the observed time series. Fitting was performed with temperature-specific $K_{i}$ as an environment-dependent parameter for each species $i$ resulting in temperature-specific $r_{i}$ values. Using these parameters, the fitting was performed to 2 -species and 3-species mixtures as well as to each replicate (see Fig. 1 as an example). We used the Nelder-Mead algorithm for optimizing the mean absolute error (MAE) between observations and predictions.

The model selection was based on maximizing the partial correlation between the fitted and observed time series data (controlling for time). We selected the simplest model (with the lowest $\beta$ ) from a $5 \%$ deviation interval from the highest partial correlation coefficient. This procedure resulted in the selection of the linear LV model $77 \%$ of cases for 2 -species mixtures and $51 \%$ of cases for 3 -species mixtures. Note that $r_{i}$ and $K_{i}$ are inferred from monocultures, we set $a_{i i}=1$ in consistency with the K-formalism ${ }^{26}$, and all cases yield topologically similar models to the LV model ${ }^{29}$. We also tested the robustness of this relationship by bootstrapping the time series 100 times using a uniform sampling within $\pm 1 \%$ of each data point and recalculating all our measures from these slightly perturbed time series. This sensitivity analysis provided appropriate confidence intervals for each variation and regression coefficient given that observational noise is unavoidable.

\section{Data and code availability}

The experimental data used in this study are available as indicated in Ref. ${ }^{39}$. Codes for Figs. 2-3 are available at https://github.com/MITEcology/NEE_Tabi_et_al_2020.

\section{Acknowledgements}

The University of Zurich Research Priority Programme on Global Change and Biodiversity supported this research. Furthermore, funding came from the Swiss National Science Foundation (grant 31003A_159498 to OP). Funding was also provided by the Mitsui Chair (SS). 
336 This is also publication ISEM-2020-075 of the Institut des Sciences de l'Evolution - Montpellier

337 (EAF). We thank Yves Choffat, Pravin Ganesanandamoorthy, Aurelie Garnier, Jason I.

338 Griffiths, Suzanne Greene, Thomas M. Massie, Gian Marco Palamara and Mathew Seymour for

339 help with the data collection. We also thank Mohammad AlAdwani, Simone Cenci, and

340 Chuliang Song for insightful discussions about this study.

\section{${ }_{341}$ Contributions}

342 A.T. and S.S. conceived and wrote the study, analyzed and interpreted the data. O.P. took part 343 in reviewing and editing process. Other authors contributed to the experiment from which data 344 is used as stated in Pennekamp et al $(2018)^{39}$.

\section{Competing interests}

346 The authors declare no competing interests. 


\section{References}

1. Fukami, T. Annual Review of Ecology, Evolution, and Systematics 46 (2015).

2. Cenci, S., Song, C., and Saavedra, S. Ecology and Evolution 8, 6852-6859 (2018).

3. Hutchins, L. W. Ecological Monographs 17(3), 325-335 February (1947).

4. Brown, J. H., Gillooly, J. F., Allen, A. P., Savage, V. M., and West, G. B. Ecology 85(7), 1771-1789 July (2004).

5. Tylianakis, J. M., Didham, R. K., Bascompte, J., and Wardle, D. A. Ecology Letters 11(12), 1351-1363 December (2008).

6. Jiang, L. and Morin, P. J. Journal of Animal Ecology 73(3), 569-576 May (2004).

7. Kordas, R. L., Harley, C. D. G., and O'Connor, M. I. Journal of Experimental Marine Biology and Ecology 400(1), 218-226 April (2011).

8. O'Connor, M. I., Piehler, M. F., Leech, D. M., Anton, A., and Bruno, J. F. PLOS Biol 7(8), e1000178 August (2009).

9. Petchey, O. L., McPhearson, P. T., Casey, T. M., and Morin, P. J. Nature 402(6757), 69-72 November (1999).

10. Sentis, A., Hemptinne, J.-L., and Brodeur, J. Ecology Letters 17(7), 785-793 July (2014).

11. Wootton, J. T. Annual Review of Ecology and Systematics 25, 443-466 (1994).

12. Bruno, J. F., Stachowicz, J. J., and Bertness, M. D. Trends in Ecology $\&$ Evolution 18(3), 119-125 March (2003).

13. Koltz, A. M., Classen, A. T., and Wright, J. P. Proceedings of the National Academy of Sciences 115(32), E7541-E7549 August (2018).

14. Song, C., Ahn, S. V., Rohr, R. P., and Saavedra, S. Trends in Ecology 8 Evolution in press, No. 2641 (2020).

15. Montoya, J., Woodward, G., Emmerson, M. C., and Solé, R. V. Ecology 90(9), 2426-2433 September (2009).

16. Higashi, M. and Patten, B. C. The American Naturalist 133(2), 288-302 (1989).

17. Binzer, A., Guill, C., Brose, U., and Rall, B. C. Phil. Trans. R. Soc. B 367(1605), 2935-2944 November (2012). 
18. Binzer, A., Guill, C., Rall, B. C., and Brose, U. Global Change Biology 22(1), 220-227 January (2016).

19. Sentis, A., Binzer, A., and Boukal, D. S. Ecology Letters 20(7), 852-862 July (2017).

20. Dell, A. I., Pawar, S., and Savage, V. M. Proceedings of the National Academy of Sciences of the United States of America 108(26), 10591-10596 June (2011).

21. Davis, A. J., Jenkinson, L. S., Lawton, J. H., Shorrocks, B., and Wood, S. Nature 391(6669), 783-786 February (1998).

22. Allison, S. D., Lu, Y., Weihe, C., Goulden, M. L., Martiny, A. C., Treseder, K. K., and Martiny, J. B. H. Ecology 94(3), 714-725 March (2013).

23. Widder, S., Allen, R. J., Pfeiffer, T., Curtis, T. P., Wiuf, C., Sloan, W. T., Cordero, O. X., Brown, S. P., Momeni, B., Shou, W., Kettle, H., Flint, H. J., Haas, A. F., Laroche, B., Kreft, J.-U., Rainey, P. B., Freilich, S., Schuster, S., Milferstedt, K., Meer, J. R. v. d., Grokopf, T., Huisman, J., Free, A., Picioreanu, C., Quince, C., Klapper, I., Labarthe, S., Smets, B. F., Wang, H., Fellows, I. N. I., and Soyer, O. S. The ISME Journal 10(11), 2557-2568 November (2016).

24. Saavedra, S., Rohr, R. P., Bascompte, J., Godoy, O., Kraft, N. J. B., and Levine, J. M. Ecological Monographs 87(3), 470-486 August (2017).

25. Song, C., Rohr, R. P., and Saavedra, S. Journal of Theoretical Biology 450, 30-36 August (2018).

26. Vandermeer, J. H. Science 188(4185), 253-255 (1975).

27. Rohr, R. P., Saavedra, S., Peralta, G., Frost, C. M., Bersier, L.-F., Bascompte, J., and Tylianakis, J. M. The American Naturalist August (2016).

28. Logofet, D. O. Stability Problems in Mathematical Ecology, CRC, Boca Ratón, FL (1993).

29. Cenci, S. and Saavedra, S. Physical Review E 97(1), 012401 January (2018).

30. Saavedra, S., Rohr, R. P., Gilarranz, L. J., and Bascompte, J. J. R. Soc. Interface 11, 20140693 (2014).

31. Altermatt, F., Fronhofer, E. A., Garnier, A., Giometto, A., Hammes, F., Klecka, J., Legrand, D., Mächler, E., Massie, T. M., Pennekamp, F., Plebani, M., Pontarp, M., Schtickzelle, N., Thuillier, V., and Petchey, O. L. Methods in Ecology and Evolution 6(2), 218-231 February (2015). 
32. Fox, J. W. and Morin, P. J. Journal of Animal Ecology 70(1), 80-90 January (2001).

33. Van der Putten, W. H., Macel, M., and Visser, M. E. Philosophical Transactions of the Royal Society B: Biological Sciences 365(1549), 2025-2034 July (2010).

34. Song, C. and Saavedra, S. Ecology 99(3), 743-751 December (2017).

35. Cottingham, K. L., Lennon, J. T., and Brown, B. L. Frontiers in Ecology and the Environment 3(3), 145-152 (2005).

36. Leary, D. J. and Petchey, O. L. Journal of Animal Ecology 78(6), 1143-1151 November (2009).

37. Pennekamp, F., Schtickzelle, N., and Petchey, O. L. Ecology and Evolution 5(13), 2584-2595 July (2015).

38. Pennekamp, F., Griffiths, J. I., Fronhofer, E. A., Garnier, A., Seymour, M., Altermatt, F., and Petchey, O. L. PLOS ONE 12(5), e0176682 May (2017).

39. Pennekamp, F., Pontarp, M., Tabi, A., Altermatt, F., Alther, R., Choffat, Y., Fronhofer, E. A., Ganesanandamoorthy, P., Garnier, A., Griffiths, J. I., Greene, S., Horgan, K., Massie, T. M., Mächler, E., Palamara, G. M., Seymour, M., and Petchey, O. L. Nature 563(7729), 109 November (2018). 

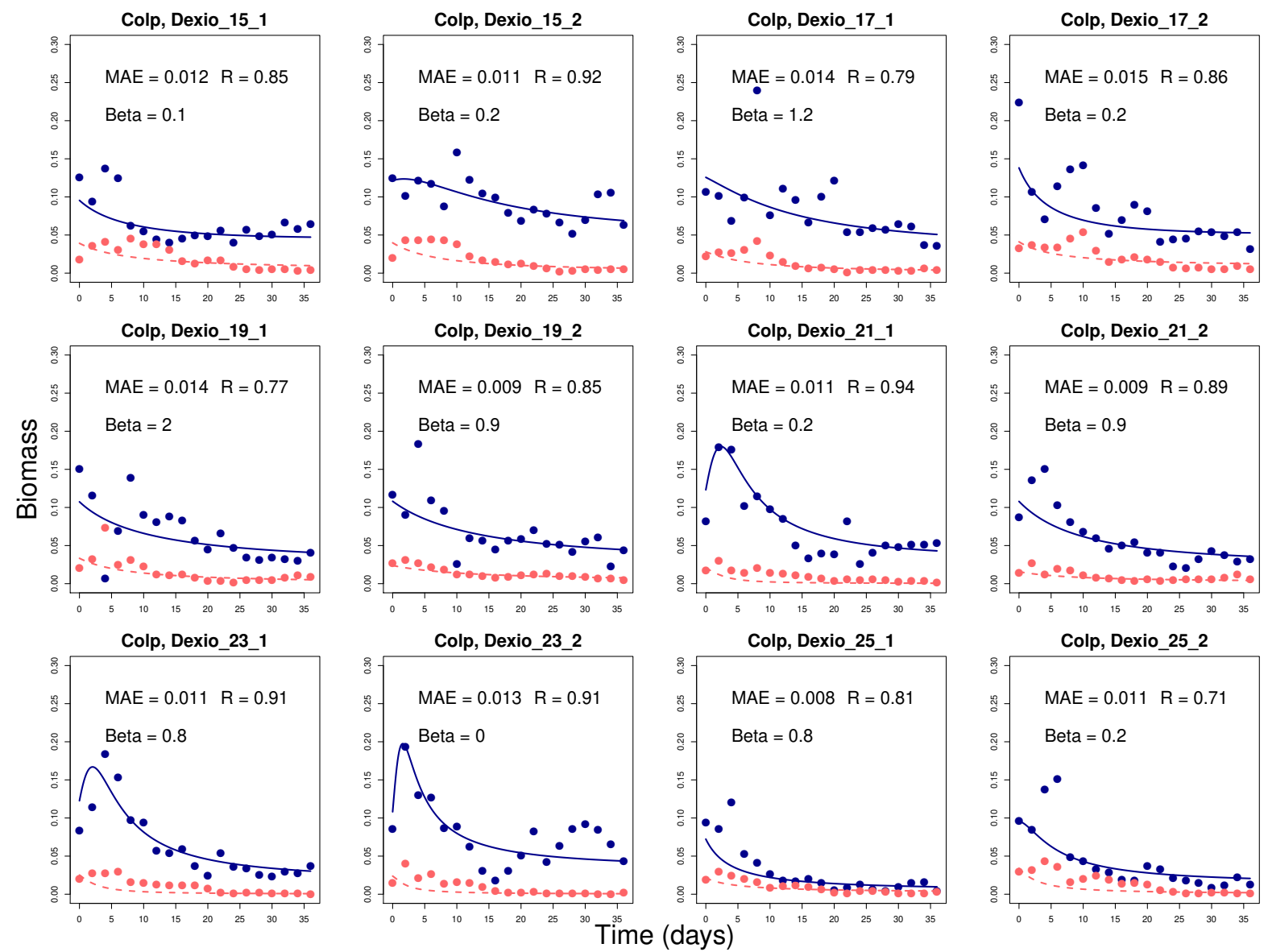

Figure 1: Example of fitting 2-species GLV model across temperature. Illustration using time series of interacting Colpidium (blue) and Dexiostoma (red) as an example. Each panel shows a different temperature-replicate combination. Dots are the observations and the corresponding lines indicate the prediction of the best fitting model. The mean absolute error (MAE), partial correlation $(\mathrm{R})$ and the tuning parameter $(\beta)$ of the best fit are also plotted in each graph. 

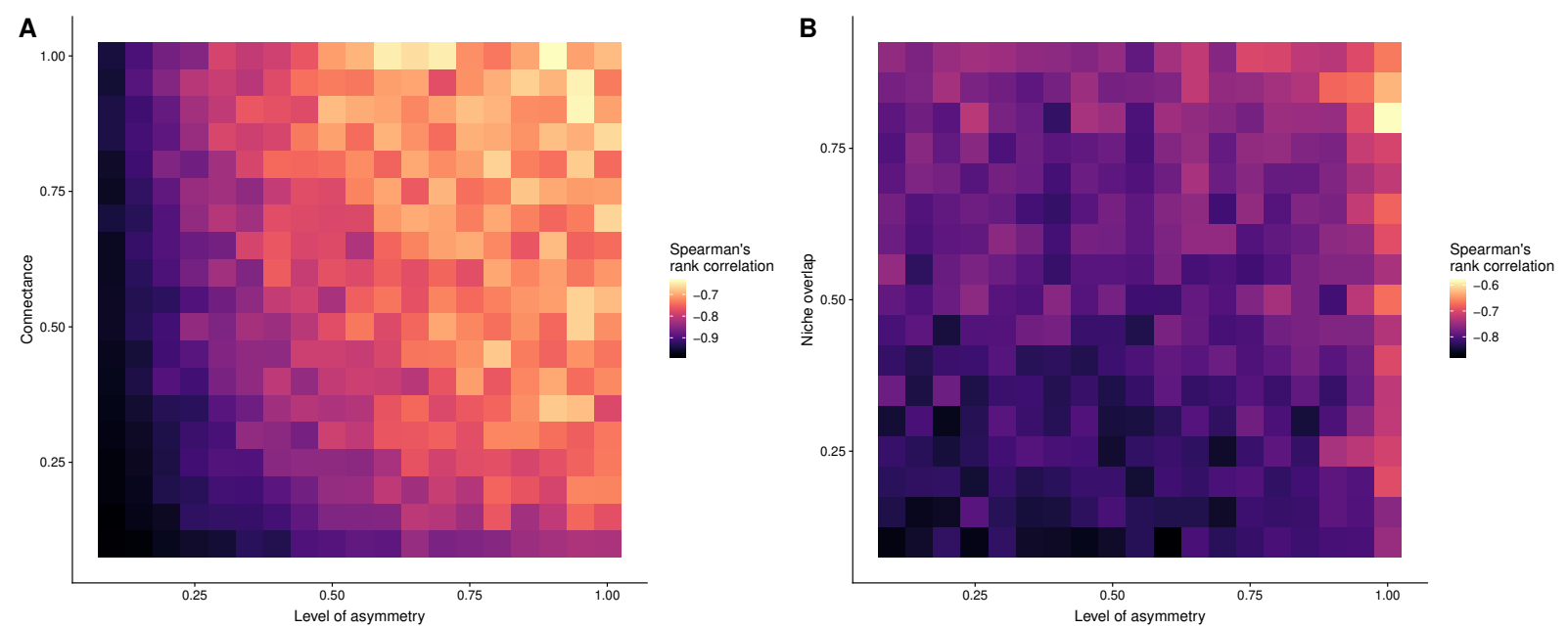

Figure 2: The effect of $(\mathbf{A})$ connectance, $(\mathbf{B})$ niche overlap and asymmetry on the relationship (measured as the Spearman's rank correlation) between species evenness and relative performance in isolation in 10-species communities. Panel (A) shows a strong interaction between asymmetry and connectance, i.e. high asymmetry and connectance leads to the weaker negative correlation between species evenness and the relative performance in isolation. Connectance is measured as the fraction of non-zero coefficients and modeled following Ref. ${ }^{34}$. Note that the value of asymmetry corresponds to the tuning parameter $P$ used in the sampling of the interaction matrix (see Methods). In panel (B), we generated the interaction matrices based on a niche framework ${ }^{27}$, where all interaction coefficients are negative (competitive). Here, similarly to panel (A) high asymmetry and niche overlap lead to the weakest correlation.

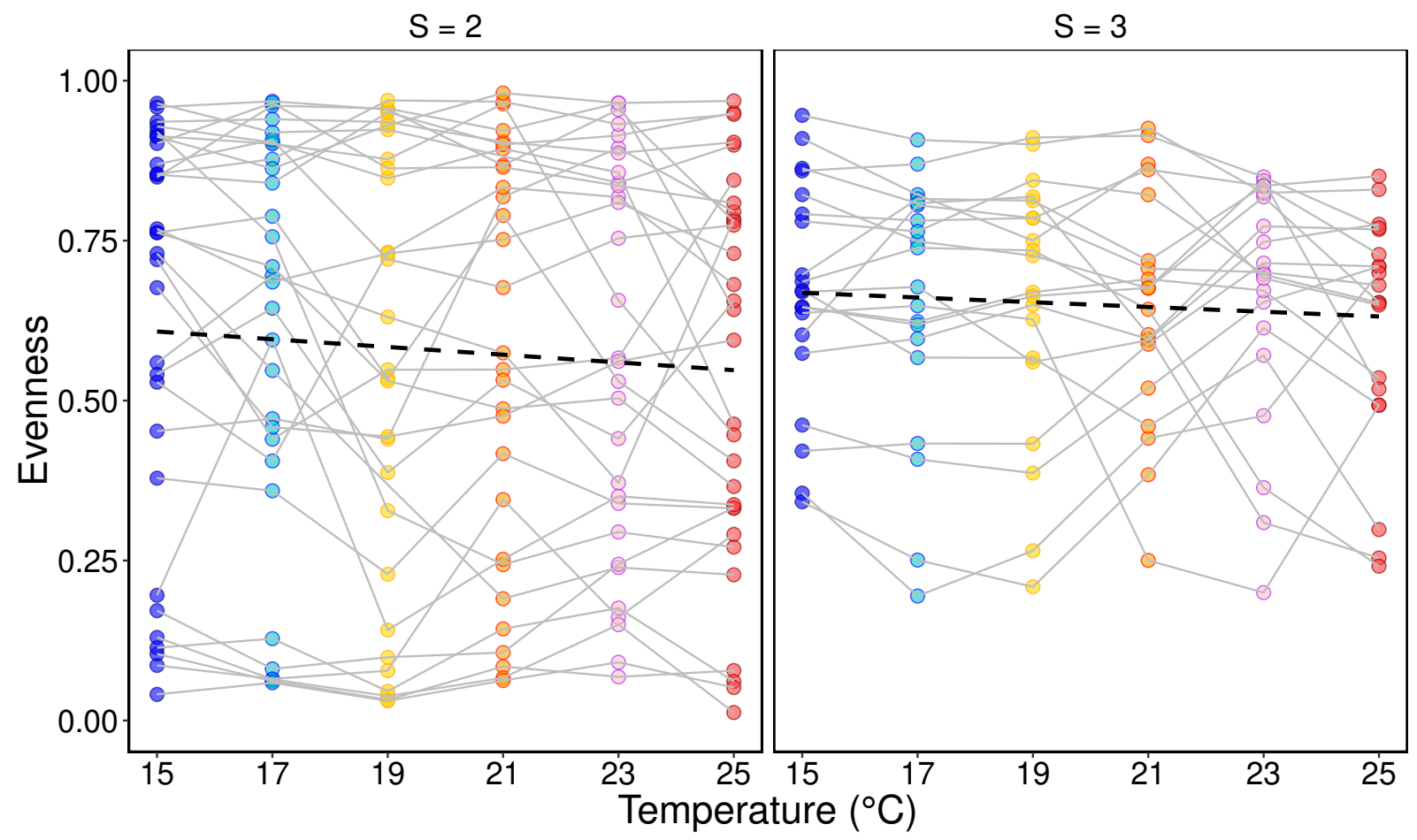

Figure 3: The relationship between species evenness and temperature empirically measured in 2and 3-species microbial communities. Species evenness was measured as the median evenness of the time series for each community. There was no statistical relationship found between species evenness and temperature. 


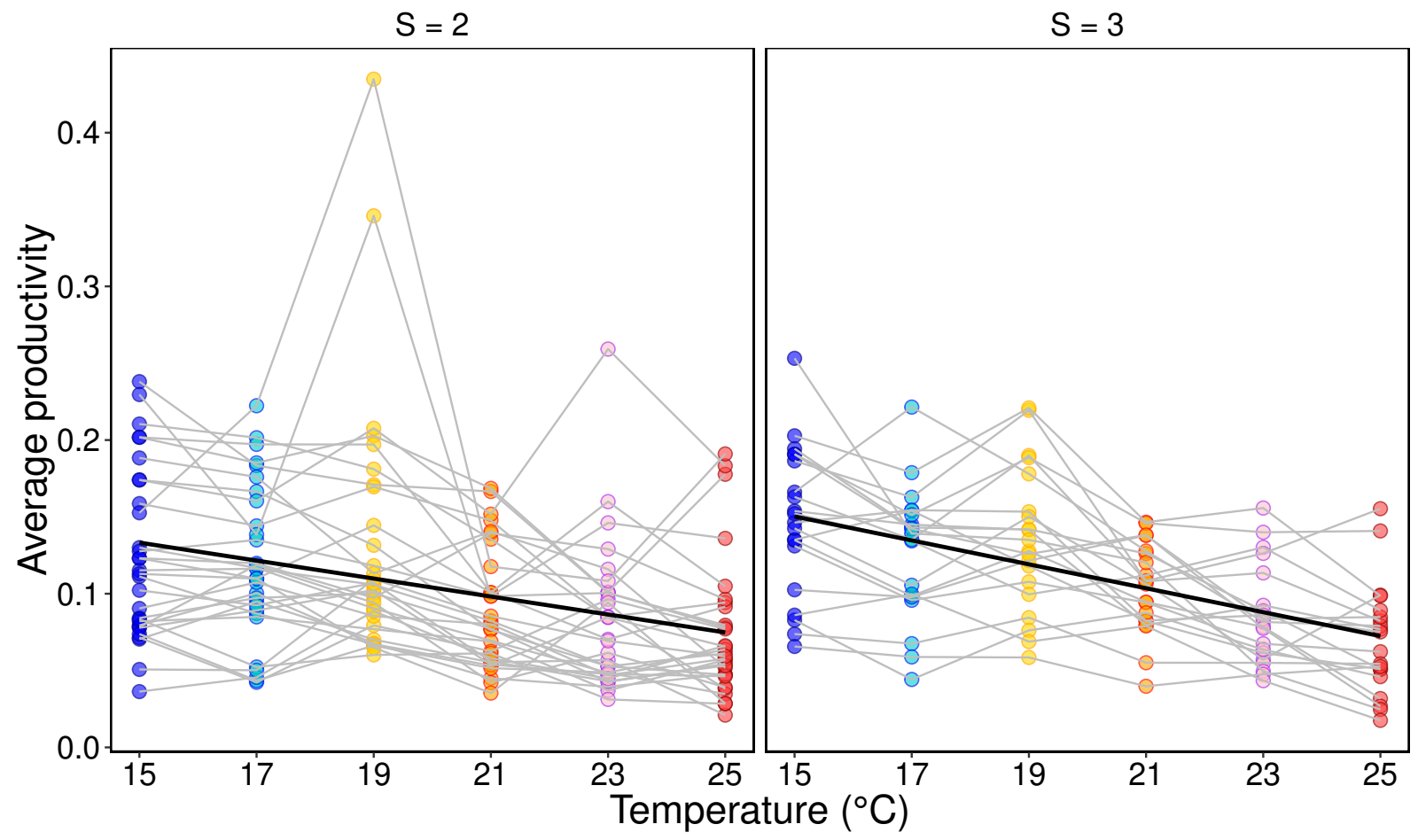

Figure 4: The relationship between average productivity and temperature empirically measured in 2 - and 3-species microbial communities. Average productivity was measured as the median of the time series of total biomass for each community. Average productivity declined with increasing temperature in 2- and 3-species communities as well. 